\title{
Bases des recommandations vaccinales
}

> Le processus d'élaboration des recommandations vaccinales, fondé sur l'établissement de la balance entre les bénéfices et les risques, est un processus complexe et continu. II fait appel à une expertise largement multidisciplinaire, prenant en compte des facteurs épidémiologiques, immunologiques, mais aussi sociaux, économiques et politiques. II repose essentiellement sur des données épidémiologiques mais fait de manière croissante appel à des outils sophistiqués de modèlisation mathématique et d'évaluation médico-économique. Les décisions doivent intégrer des concepts mal définis tels que le prix accordé par notre société à la prévention d'un décès d'enfant ou plus largement à une année de vie sauvée, les niveaux de risque d'effets secondaires et de maladies acceptables ou l'aspect collectif de la stratégie vaccinale interférant avec la liberté de choix individuelle. <

\section{Spécificités de la politique vaccinale}

Comme tout médicament et selon une procédure similaire, les vaccins sont soumis à une procédure d'autorisation de mise sur le marché (AMM) permettant d'en garantir la qualité, la sécurité et l'efficacité. L'obtention de I'AMM pour un vaccin permet au producteur de commercialiser le produit, considéré comme suffisamment sûr et efficace. Cependant, elle ne donne pas d'indication sur l'intérêt du produit comme outil d'une stratégie de santé publique, dans le cadre d'une politique de prévention des maladies infectieuses. En effet, à la différence du champ du médicament, l'acte vaccinal n'est pas dicté par une situation morbide individuelle rendant nécessaire la mise en œuvre d'une thérapeutique spécifique. Outil de prévention primaire, la vaccination cible des sujets sains et s'inscrit le plus souvent dans une logique collective, où la démarche vaccinale est renforcée par la possibilité, si un niveau élevé de couverture est atteint, de maîtriser un agent infectieux, en particulier pour les pathologies

Article reçu le 14 décembre 2006, accepté le 11 février 2007.

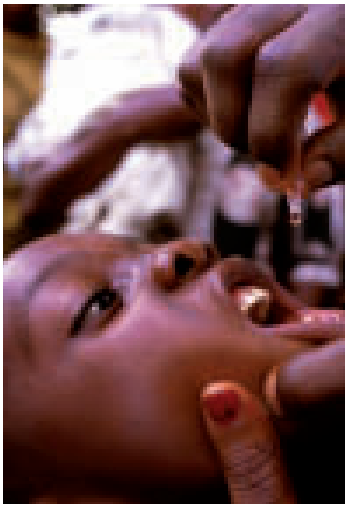

Institut de Veille Sanitaire, Département des maladies infectieuses, 12, rue du Val d'Osne, 94415 Saint-Maurice Cedex, France. d.levybruhl@invs.sante.fr

à transmission strictement inter-humaine. Pour de telles maladies, la diminution du nombre de cas induite par la vaccination se traduit par une diminution du nombre de sources potentielles de contamination et donc du risque d'infection pour les sujets non vaccinés vivant au sein d'une population bien vaccinée. Ce risque peut même disparaître si la couverture vaccinale est suffisamment élevée pour qu'à un moment donné tous les cas quittent la phase infectieuse (définie comme la période durant laquelle un malade est contagieux) sans avoir rencontré un sujet réceptif. La maladie est alors éliminée du pays, voire, si ce mécanisme est mis en jeu simultanément dans l'ensemble du monde, éradiquée. C'est ainsi que la vaccination est la seule intervention de santé publique qui ait permis de faire disparaître complètement une maladie: il s'agit de la variole, dont l'éradication a été certifiée par l'OMS en 1980. Le recul disponible aujourd'hui permet d'affirmer que le virus a totalement disparu de la surface du globe, à l'exception des laboratoires où il est encore détenu, légalement ou non.

Cependant les effets indirects de réduction de la circulation d'un agent pathogène ne sont pas toujours favorables. Pour ces mêmes maladies à transmission strictement inter-humaine, un niveau de couverture vaccinale élevé, mais insuffisant pour permettre l'élimination de la maladie, peut être préjudiciable. La réduction du risque d'infection pour les sujets non vaccinés vivant au sein d'une population bien vaccinée va leur permettre de grandir plus longtemps sans rencontrer un cas susceptible de les contaminer. Si le niveau résiduel de circulation de l'agent infectieux est 
suffisant pour qu'ils finissent par être un jour au contact d'un cas, l'âge moyen auquel ils feront la maladie sera plus élevé qu'à l'ère pré-vaccinale. Pour une maladie dont la gravité augmente avec l'âge de survenue, les cas seront donc plus souvent sévères.

Un autre effet indirect d'un programme de vaccination peut survenir lorsque le vaccin ne protège que contre un nombre limité des sérotypes ou sérogroupes de l'agent pathogène. Le risque existe d'une compensation de la diminution des formes liées aux sérotypes ou sérogroupes inclus dans le vaccin par une augmentation des formes non couvertes par le vaccin. Au mieux, la conséquence en serait la diminution de l'impact épidémiologique attendu de la vaccination. Au pire, d'un point de vue théorique, rien n'interdit d'imaginer que la vaccination pourrait sélectionner des sérotypes ou sérogroupes mineurs qui pourraient s'avérer plus pathogènes que ceux inclus dans le vaccin [1].

\section{Processus décisionnel en matière de vaccination}

La prise en compte, au-delà des effets directs de protection des sujets vaccinés, des effets épidémiologiques indirects de la vaccination, qu'ils soient favorables ou défavorables, est au cœur du processus d'élaboration des recommandations vaccinales par le Comité technique des Vaccinations (CTV). Ce comité d'experts était, jusqu'à la fin 2006, un sous-groupe permanent de la section des maladies transmissibles du Conseil supérieur d'hygiène publique de France (CSHPF), groupe d'experts auprès du ministère de la Santé, chargé de lui faire des recommandations sur les modalités de lutte contre les maladies. Le CTV est maintenant rattaché à la Commission spécialisée Sécurité sanitaire du Haut conseil de la santé publique (HCSP). Il est composé de médecins infectiologues, de pédiatres, de microbiologistes, de représentants des Centres nationaux de référence, de médecins de santé publique et d'épidémiologistes. Depuis le début des années 2000, y siègent en outre un médecin généraliste, un médecin du travail et un immunologiste. À côté des membres de ce comité, seuls habilités à prendre part aux votes, des représentants des agences de l'État, des directions des ministères impliqués, ainsi que des invités permanents participent aux travaux du comité. À l'occasion de la mise sur le marché d'un nouveau vaccin, un avis est préparé par un groupe de travail ad hoc, en faveur d'une vaccination de routine d'une ou plusieurs cohortes d'enfants ou d'adultes, d'une recommandation limitée à des populations ciblées, ou de l'absence d'intégration dans le calendrier vaccinal. Une fois adopté, le cas échéant après modifications, l'avis est soumis pour approbation au CSHPF/ HCSP. Si l'avis est adopté, il est transmis au ministère chargé de la Santé. Les nouveaux avis, entérinés par le Ministère, sont publiés dans son Bulletin Officiel. Ils constituent la base de l'actualisation du calendrier vaccinal qui donne lieu à une publication annuelle dans le Bulletin Épidémiologique Hebdomadaire $(\mathrm{BEH})$, largement reprise dans la presse médicale.

\section{Paramètres intervenant dans la décision vaccinale}

La très large multidisciplinarité de ce comité lui permet de prendre en compte, dans l'élaboration et l'adaptation du calendrier vaccinal, les différents déterminants de la décision vaccinale. II s'agit essentiellement de:

- L'épidémiologie de la maladie. Les paramètres les plus importants sont son poids en terme de morbidité, de mortalité et de séquelles graves ainsi que l'identification des populations les plus à risque. II s'agit le plus souvent de popula- tions définies en fonction de leur âge, de leur profession ou de leur mode de vie.

- L'efficacité du vaccin pour les différentes populationscibles potentielles. La connaissance de ces deux premiers éléments permet d'estimer le volet «bénéfice » attendu de la balance bénéfice/risque de la vaccination, éventuellement selon différents scénarios d'introduction dans le calendrier vaccinal.

- Les données disponibles concernant le profil de tolérance du vaccin, déterminant en grande partie le volet «risque ».

- Les modifications attendues de l’épidémiologie de la maladie induites par la vaccination. Négatives ou positives, elles interviennent dans le volet «risque» ou «bénéfice» de la vaccination. II peut s'agir des conséquences d'une couverture vaccinale insuffisante, d'une stratégie vaccinale inadaptée ou d'une couverture par le vaccin limitée à certains sérotypes ou sérogroupes du micro-organisme responsable de la maladie. II peut s'agir au contraire d'un impact additionnel pour les sujets non vaccinés ou plus largement pour des tranches d'âge non ciblées par la vaccination, voire de la possibilité d'éliminer la maladie. Des travaux de modélisation mathématique de l'impact de la vaccination sur l'épidémiologie de la maladie permettent souvent d'anticiper de tels effets.

- Le coût anticipé du vaccin et de son administration, déterminant le volet coût des rapports coût/efficacité ou coût/ avantage.

- Les données du résumé des caractéristiques du produit (RCP) concernant les modalités d'administration ayant fait l'objet de I'AMM (âge d'administration, nombre de doses, nécessité d'un rappel, possibilité d'associations avec les autres vaccins...).

- La faisabilité de l'intégration dans le calendrier vaccinal au regard des vaccinations déjà incluses et des contraintes organisationnelles des services de santé.

- La pertinence d'une vaccination limitée à des situations épidémiologiques particulières (vaccination en post-exposition, c'est-à-dire après contact avec un cas ou en situation épidémique).

- L'existence d'autres mesures de lutte contre la maladie.

- La perception sociale de la maladie du point de vue du corps médical et du public.

- Les objectifs de maîtrise de la maladie par la vaccination, définis au niveau international.

\section{Contexte actuel de la décision vaccinale}

L'analyse de la situation actuelle concernant certains de ces paramètres peut conduire aux constats suivants.

\section{Bénéfice attendu des nouveaux vaccins}

Les nouveaux vaccins qui sont proposés correspondent le plus souvent à des maladies qui ne sont plus les fléaux infectieux 
qu'étaient, dans la première partie du siècle dernier, des maladies telles que la diphtérie, la poliomyélite, la coqueluche ou le tétanos. Les bénéfices attendus en termes de morbidité sévère ou de mortalité évitées sont donc le plus souvent limités. À l'exception des vaccins contre les papillomavirus humains, le nombre de décès évitables chaque année en France par les vaccins récemment mis sur le marché, tels que les vaccins contre le rotavirus, contre la varicelle ou contre les méningites à pneumocoque ou méningocoque se compte au mieux en quelque dizaines, d'après les estimations effectuées par l'Institut de veille sanitaire. Le nombre de décès évitables chaque année par les vaccins contre la diphtérie, la coqueluche ou le tétanos se comptaient chaque année en centaines [2].

\section{Coût des nouveaux vaccins}

Ces nouveaux vaccins, faisant souvent appel aux technologies les plus récentes, sont le fruit d'efforts de recherche de plus en plus coûteux. Le coût très élevé de leur développement est également le reflet des exigences croissantes des autorités d'enregistrement en matière de sécurité, imposant, de la part des producteurs, des investissements de plus en plus importants pour y répondre [3].

Ces deux points aboutissent à des conflits potentiels entre des produits que les industries du vaccin vont proposer à des prix élevés et un intérêt de santé publique, mesuré en termes de morbidité sévère et de mortalité évitables, limité. Cependant, cet impact épidémiologique limité peut, pour des maladies très fréquentes, être en partie compensé par l'intérêt économique pour la société de réduire les dépenses liées aux arrêts de travail pris par les sujets malades ou à l'occasion de la maladie d'un enfant. Ainsi la vaccination des nourrissons contre la varicelle, maladie exceptionnellement sévère, s'est avérée dans la grande majorité des études, susceptible d'engendrer des économies pour la société [4].

\section{Profil de tolérance réel ou perçu}

À des degrés divers, la sensibilité des sociétés vis-à-vis de la sécurité des vaccins s'est accrue dans les dernières décennies, dans l'ensemble du monde industrialisé. Cette sensibilité s'exerce aux niveaux des différents acteurs de la politique vaccinale:

\section{- Au niveau des décideurs}

Les autorités de santé publique sont parfois réticentes à intégrer de nouveaux produits dans le calendrier vaccinal, de par la sensibilité croissante de nos sociétés vis-à-vis des effets secondaires des vaccins. Que l'association entre la vaccination et l'effet secondaire existe ou non, plus l'utilisation du vaccin sera large, plus le nombre d'effets secondaires survenant dans les suites d'une vaccination augmentera, ne serait-ce que par simple association temporelle. La crainte de se voir reprocher des effets secondaires, réels ou supposés, peut être un frein à une large recommandation vaccinale.

\section{- Au niveau d'une certaine proportion du corps médical et de la population}

D'une manière qui peut paraître paradoxale, les réticences vis-à-vis de l'acte vaccinal semblent augmenter, alors que, comme déjà mentionné, la sécurité des vaccins a été largement améliorée par les nouvelles techniques de production et que les exigences des autorités d'enregistrement de nouveaux produits n'ont jamais été aussi élevées. Cette situation reflète probablement en grande partie le fait que les vaccins nouvellement introduits, comme ceux contre la rougeole, la rubéole et les oreillons ou contre l'hépatite $B$, ont été perçus, et jusqu'à un certain point à juste titre, comme protégeant contre des maladies qui ne repré- sentaient pas un danger de la même ampleur que celles contre lesquelles luttaient les vaccins plus anciens. Ces nouveaux vaccins ont également pâti, dans une certaine mesure, du succès des vaccinations plus anciennes, qui en faisant disparaître les maladies-cibles, ont fait oublier les dangers représentés par les maladies infectieuses. Cette situation témoigne peut-être également d'une évolution de nos sociétés vers plus d'individualisme dans la gestion de la décision de soins. La décision vaccinale est davantage vécue comme le fruit d'un arbitrage individuel entre les risques, consentis, de la maladie et ceux, perçus comme subis, de la vaccination. Elle ne veut plus résulter de l'application systématique d'une décision prise à l'échelle de la collectivité, sur la base d'une expertise concluant à une balance bénéfice/ risque favorable à la vaccination, qui n'est plus perçue comme infaillible.

La balance bénéfice/risque d'une vaccination constitue en effet, comme pour toute intervention de santé publique, en particulier en matière de prévention, le concept de base qui sous-tend la décision. C'est également le critère implicite auquel se réfère chaque individu, confronté pour lui-même ou son enfant, à une décision en matière vaccinale. Cependant, si, au niveau de la population, les paramètres de cette équation peuvent être quantifiés à travers des valeurs moyennes attendues (nombre moyen de décès évités versus nombre moyen d'effets secondaires sévères attendus), il n'en est pas de même au niveau individuel. La comparaison devrait se faire en fonction de la probabilité de réalisation de chacun des deux risques, celui de la survenue de la maladie évitable par la vaccination, et celui de la survenue d'un effet secondaire sévère en cas de vaccination. Or une telle comparaison est extrêmement difficile à appréhender, dès lors que les probabilités de survenue de ces deux risques sont très faibles. Des mécanismes de type cognitifs interférent avec la rationalité de la décision: le risque vaccinal est immédiat, alors que le bénéfice vaccinal est incertain et plus lointain. La maladie si elle survient, pourra être vécue comme le fruit de la fatalité, alors que le risque vaccinal pourra être considéré comme la conséquence d'un produit défectueux ou d'une décision inconsidérée des autorités de santé publique. L'histoire récente de la vaccination conte l'hépatite $B$ en France illustre les limites, en pratique, de l'analyse bénéfice/risque.

Les bénéfices collectifs de la vaccination sont, de plus, parfois perçus comme en opposition au bénéfice individuel. Une certaine perception que ce bénéfice indirect collectif peut se faire au détriment du bénéfice direct apporté au sujet vacciné induit une suspicion envers la décision vaccinale. Il s'agit d'une idée fausse, le bénéfice collectif devant être perçu comme venant s'ajouter à la somme des bénéfices individuels. Cette affirmation souffre de très rares exceptions, dans le cas des vaccinations actuellement mises en œuvre. II s'agit essentiellement de la vaccination contre la rubéole des nourrissons de sexe masculin, dont 
l'objectif est essentiellement de réduire le risque de contamination des femmes enceintes en réduisant la circulation virale. Bien entendu, cette stratégie est justifiée par l'excellente tolérance du vaccin.

\section{Risques ou bénéfices additionnels}

liés à la modification de l'épidémiologie de la maladie

Le risque de survenue d'une situation épidémiologique préoccupante, si le niveau de couverture vaccinale est insuffisant, est attesté par l'expérience française et internationale concernant la vaccination contre la rougeole, la rubéole et les oreillons. Si la vaccination a permis de diminuer de manière importante l'incidence de ces trois maladies, la persistance de la circulation à un faible niveau de ces virus, associée à l'existence d'une fraction de la population qui échappe à la vaccination, conduit à la survenue de bouffées épidémiques au cours desquelles les grands enfants et les jeunes adultes sont particulièrement atteints. L'épidémie de rubéole congénitale survenue en Grèce en 1993 ou, plus récemment, les épidémies de rougeole qui ont touché plusieurs pays d'Europe en 2005 en sont deux illustrations $[5,6]$. En France, les données de surveillance issues du Réseau Sentinelles (Inserm U707), dans un contexte de stagnation de la couverture vaccinale à 2 ans autour de $85 \%$ depuis le milieu des années 1990, montrent que près de la moitié des cas de rougeole qui surviennent encore chaque année concernent des individus âgés de 10 ans et plus [7]. La réflexion menée actuellement concernant l'intégration de la vaccination contre la varicelle dans le calendrier vaccinal du nourrisson prend en compte également le risque, objectivé par les travaux de modélisation, d'une augmentation, en cas de couverture vaccinale insuffisante, du nombre de cas de varicelle chez l'adulte. Cette situation serait d'autant plus dommageable, qu'au-delà de l'augmentation du taux de complications et de létalité de la maladie avec l'âge, la varicelle peut induire, en cas de contamination pré- ou périnatale, des atteintes congénitales ou des varicelles néonatales très sévères.

À l'inverse, la décision prise en 2006 de généraliser à l'ensemble des nourrissons la vaccination anti-pneumococcique avec un vaccin heptavalent conjugué repose largement sur les données américaines qui ont mis en évidence des effets indirects majoritairement bénéfiques de la vaccination généralisée des nourrissons. Une des principales raisons de l'absence de recommandation en France de vaccination de tous les nourrissons était liée au fait que la vaccin n'inclut que 7 des 90 sérotypes connus de pneumocoque. II s'agit certes des sérotypes les plus souvent responsables de formes invasives mais un remplacement, sous la pression vaccinale, des sérotypes inclus dans le vaccin par des sérotypes non inclus dans le vaccin était craint. Les résultats publiés en 2005 par les Centers for Diseases Control (CDC) américains de l'impact de la vaccination des nourrissons mise en œuvre fin 2000 étaient, à cet égard, rassurants. Ils font même état d'un impact bien plus important de la vaccination que celui qui était attendu du seul fait de l'efficacité vaccinale directe [8]. La diminution observée en 2003 des cas d'infections invasives à pneumocoque dus à des souches de sérotype vaccinal chez les enfants de moins de 5 ans aux États-Unis était de $94 \%$. Or, la diminution attendue du fait de l'efficacité vaccinale directe du vaccin était de $64 \%$, compte tenu d'une couverture vaccinale de $68 \%$ et d'une efficacité vaccinale estimée lors des essais cliniques à $94 \%$. Un effet indirect de la vaccination des enfants était aussi observé dans des tranches d'âge non couvertes par la vaccination : les cas d'infections invasives à pneumocoque ont diminué de $28 \%$ chez les adultes de plus de 64 ans. Cette diminution portait spécifiquement sur les cas dus aux souches de sérotype vaccinal. Ces bénéfices indirects d'immunité de groupe sont attribués à la diminution du portage du pneumocoque chez les enfants vaccinés, ce qui réduirait le risque d'exposition des personnes non vaccinées. Au total, les auteurs ont estimé que, parmi les cas dus à des souches de sérotype vaccinal évités par la vaccination, $69 \%$ étaient évités par un effet indirect de la vaccination. II est important de noter que ces résultats on été obtenus malgré une augmentation modérée (+21\%) de l'incidence des cas dus à des souches de sérotype non vaccinal observée chez l'enfant de moins de 5 ans. Ce remplacement sérotypique était, dans une moindre mesure, également observé chez l'adulte.

\section{Faisabilité de l'intégration}

\section{d'un nouveau vaccin dans le calendrier vaccinal}

Parmi les craintes qui sont le plus souvent mises en avant par les parents concernant les vaccins, figure l'augmentation du nombre de vaccinations proposées aux nourrissons. Si les immunologistes s'accordent à penser qu'il n'y a aucun danger de surcharge du système immunitaire liée à l'addition d'antigènes vaccinaux [9], l'augmentation du nombre d'injections se heurte à l'acceptabilité du corps médical et des familles. Le respect des recommandations du calendrier vaccinal 2006 requiert, pour un enfant en mode de garde collectif, 14 injections vaccinales entre la naissance et l'âge de 2 ans (le BCG, 4 doses de vaccin quintuple diphtérie-tétanos-coqueluche-polio-Haemophilus influenzae $b$, deux doses de vaccins contre la rougeole, les oreillons et la rubéole, 3 injections de vaccin contre l'hépatite $B$ et 4 doses de vaccin contre les infections invasives à pneumocoque) [10].

Les possibilités de contournement de ces réticences par les combinaisons, au sein d'un même produit, de plusieurs antigènes de natures différentes sont limitées. Cela est attesté par la diminution de l'immunogénicité des valences Haemophilus influenzae b ou hépatite B lorsqu'elles sont combinées au sein de préparations hexavalentes (incluant en plus les valences diphtérie, tétanos, coqueluche et poliomyélite) [11].

\section{Existence d'autres mesures de lutte contre la maladie}

La décision vaccinale doit parfois prendre en compte l'existence d'autres interventions de santé publique avec lesquelles la vaccination doit être comparée. C'est le cas pour les nouveaux vaccins contre les infections à papillomavirus, responsables du cancer du col de l'utérus. Ainsi, malgré les données très favorables concernant l'efficacité et le profil de tolérance de ces vaccins, la question se pose de la pertinence d'une large recommandation de vaccination des jeunes filles ou des jeunes femmes, en comparaison avec une augmentation de la couverture du dépistage des lésions pré-cancereuses par frottis cervico-utérin. Des travaux de modélisation mathématique 
et d'évaluation médico-économique sont en cours en France, destinés à comparer l'impact et le coût/efficacité de différentes alternatives (maintien du dépistage à son niveau actuel et vaccination, organisation au niveau national du dépistage, organisation au niveau national du dépistage et vaccination).

\section{Adaptation de stratégies vaccinales pour des vaccins intégrés dans le calendrier vaccinal}

Une fois un vaccin intégré dans le calendrier vaccinal, un suivi de son impact épidémiologique est nécessaire, permettant le cas échéant d'adapter la stratégie vaccinale en fonction des modifications de l'épidémiologie, qu'elles soient induites par la vaccination elle-même ou par d'autres facteurs. Plusieurs exemples de modifications récentes du calendrier vaccinal français illustrent ce propos:

- Les données d'une enquête évaluant le profil immunitaire de la population française vis-à-vis de la diphtérie ont montré une protection insuffisante dans les tranches d'âge les plus âgées, ce qui a conduit en 2005 à l'introduction dans le calendrier vaccinal de rappels antidiphtériques décennaux chez l'adulte [12].

- L'analyse des données engendrées par le réseau de surveillance hospitalier de la coqueluche Renacoq a permis de montrer qu'entre un tiers et la moitié des contaminateurs des très jeunes nourrissons atteints de coqueluche, trop jeunes pour avoir bénéficié de la protection vaccinale, étaient des adultes [13]. Cette analyse a conduit à introduire dans le calendrier vaccinal 2004 une recommandation de vaccination des adultes susceptibles de devenir parents dans les mois ou années à venir ou à l'occasion d'une grossesse dans le foyer familial (voir l'article de Benjamin Silbermann et Odile Launay, page 423 de ce numéro) [14].

- La diminution d'incidence de la tuberculose a conduit à réévaluer la pertinence de la vaccination BCG systématique et obligatoire avant l'entrée en collectivité des enfants. Les conclusions de l'analyse bénéfice/risque menée étaient en faveur d'un passage à une vaccination ciblée sur les enfants à risque élevé de tuberculose [15]. La mise en œuvre de cette stratégie, adoptée dans son principe par le Comité technique des vaccinations et le Conseil supérieur d'hygiène publique de France fin 2005, a été conditionnée par ces instances au renforcement préalable des autres mesures de lutte contre la tuberculose, afin de réduire le risque d'exposition au bacille tuberculeux des enfants non vaccinés. Cependant, les difficultés opérationnelles posées par la disparition début 2006 de la multipuncture, technique qui était utilisée quasi exclusivement pour la primo-vaccination, ont conduit ces mêmes instances à proposer, début 2007, une mise en œuvre plus rapide de la vaccination BCG ciblée sur les populations d'enfants à risque. $\diamond$

\section{SUMMARY}

Basis for vaccine recommendations

Once a new vaccine has been granted its licensing, a public health expertise is needed in order to support the decision regarding its possible inclusion within the national immunisation schedule. This analysis, based on an assessment of the benefits/risks balance and costs/effectiveness ratio, is a multidisciplinary exercise. Largely based on epidemiological and immunological expertises, it also requires biomathematical and economical inputs, if the long term consequences of the vaccination are to be taken into account. Indeed, the main drivers of the decision are the burden of the disease, the characteristics of the vaccine in term of effectiveness and safety, the cost of the vaccination, the feasibility of the adjunction of the vaccine in the schedule, the social demand for this vaccination and the positive or negative indirect effects of a large vaccination on the epidemiology of the disease, in addition to the direct protective effect for vaccinated individuals. New vaccines are generally characterised by a more limited epidemiological impact than older vaccines, in a context of growing requirements from our society regarding drugs, and especially vaccines, safety. Both the real and perceived benefits/risks balances for the more recent vaccines appear questionable. The possibility of detrimental epidemiological consequences of either insufficient vaccination coverage or serotype (or serogroup) replacement is another factor that makes the decision regarding vaccination strategies increasingly complex. $\diamond$

\section{RÉFÉRENCES}

1. Lipsitch M. Vaccination and serotype replacement. Adaptive dynamics of infectious diseases. New York: Cambridge University Press, 2002 : 362-74.

2. Vaccinations. Actualités et perspectives. Expertise Collective Inserm. Paris : Éditions Inserm, 1999: 8.

3. Rappuoli R, Miller HI, Falkow S. The intangible value of vaccination. Science $2002 ; 297: 937-9$.

4. Thiry N, Beutels P, Van Damme P, Van Doorslaer $\varepsilon$. $\varepsilon$ conomic evaluations of varicella vaccination programmes: a review of the literature. Pharmacoeconomics $2003 ; 21: 13-38$.

5. Panagiotopoulos T, Antoniadou I, Valassi-Adam $\varepsilon$. Increase in congenital rubella occurrence after immunisation in Greece: retrospective survey and systematic review. Br Med J 1999 ; 319 : 1462-7.

6. Siedler A, Tisher A, Mankertz A, Santibanez S. Two outbreaks of measles in Germany 2005. Euro-Surveillance 2006 ; 11 : 131-4.

7. Bonmarin I, Lévy-Bruhl D. Measles in France: the epidemiological impact of suboptimal immunisation coverage. Euro-Surveillance $2002 ; 7: 55-60$.

8. Centers for Diseases Control. Direct and indirect effects of routine vaccination of children with 7 -valent pneumococcal conjugate vaccine on incidence of invasive pneumococcal disease-United States, 1998-2003. Morb Mortal Wkly Rep 2005 ; 54 : 893-7.

9. Offit PA, Quarlest J, Gerber MA, et al. Addressing parents' concerns : do multiple vaccines overwhelm or weaken the infant's immune system. Pediatrics $2002 ; 209: 124-9$.

10. Calendrier vaccinal 2006. Bull Epidemiol Hebd 2006 ; 29-30:211-26.

11. Schmitt $H J$, Knuf $M, O$ rtiz $\varepsilon$, et al. Primary vaccination of infants with diphtheria-tetanus-acellular pertussis-hepatitis $B$ virus-inactivated polio virus and Hæmophilus influenzæ type b vaccines given as either separate or mixed injections. J Pediatr $2000 ; 137: 304-12$.

12. Edmunds WJ, Pebody RG, Aggerback H, et al. The sero-epidemiology of diphtheria in Western Europe. Epidemiol Infect $2000 ; 125: 113-25$.

13. Évaluation de l'impact a priori de nouvelles stratégies vaccinales. Exemples de la vaccination contre la varicelle des adolescents et contre la coqueluche des jeunes adultes. Rapport InVS, juin 2006. Saint-Maurice : Institut National de Veille Sanitaire, 2006.

14. Silbermann $B$, Launay 0 . Prévention des infections à papillomavirus et du zona: nouveaux vaccins. Med Sci (Paris) $2007 ; 23: 423-7$.

15. Lévy-Bruhl D. Estimation of the epidemiological impact of various $B C G$ vaccination scenarios in France. Rev Epidemiol Sante Publ 2005 ; 53 : 501-8.

D. Lévy-Bruht 